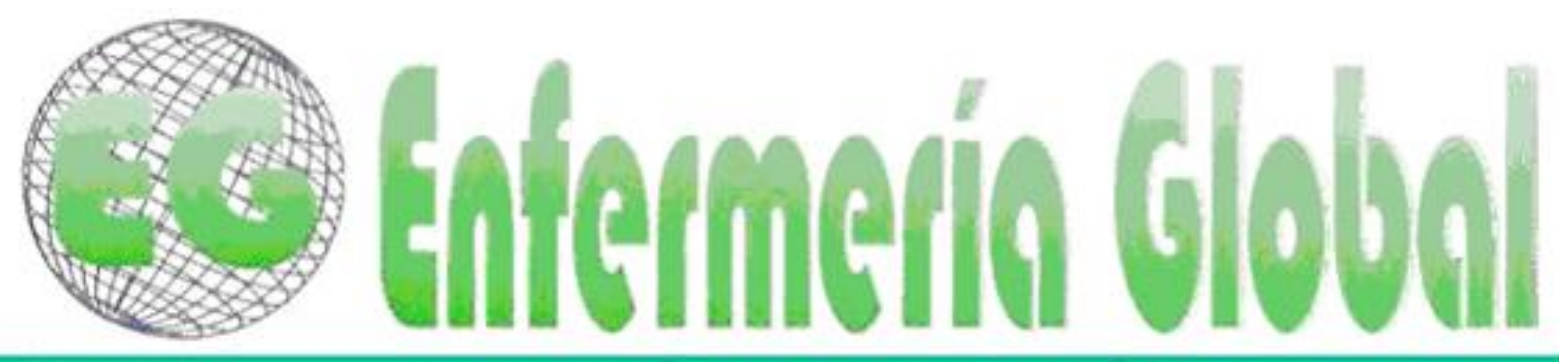

$N^{\circ} 35$

Revista electrónica trimestral de Enfermeria

Julio 2014

www.um.es/egloball

CLÍNICA

\title{
Cuidado de los pies: conocimiento de los individuos con diabetes mellitus
}

Cuidados com os pés: o conhecimento de indivíduos com diabetes mellitus

Feet care: the knowledge of individuals with diabetes mellitus

${ }^{*}$ Rodrigues Helmo, Fernanda *Dias, Flávia Aparecida *Zuffi, Fernanda Bonato ${ }^{* *}$ Borges, Maria de Fátima ${ }^{* *}$ Lara, Beatriz Hallal Jorge ${ }^{*}$ Ferreira, Lúcia Aparecida

*Curso de Graduação em Enfermagem. E-mail: lap2ferreira@yahoo.com.br ***Disciplina de Endocrinologia. Universidade Federal do Triângulo Mineiro. Uberaba, Minas Gerais, Brasil.

\begin{abstract}
Palabras clave: Diabetes Mellitus; Autocuidado; Educación en Salud
Palavras chave: Diabetes Mellitus; Autocuidado; Educação em Saúde

Keywords: Diabetes Mellitus; Self Care; Health Education.
\end{abstract}

\section{RESUMEN}

El objetivo de esta investigación fue identificar el conocimiento de las personas con diabetes mellitus en relación con el cuidado de los pies, la correlación de tiempo de estudio con el número de respuestas concordantes, así como el momento del diagnóstico de la presencia de complicaciones crónicas.

Es un estudio analítico, exploratorio y transversal realizado con 41 individuos con diabetes de una clínica de la ciudad de Triangulo Mineiro.

Los resultados mostraron un déficit en el conocimiento de esta población sobre el cuidado de los pies, ya que la puntuación media fue de 10,0 del total de 15 preguntas. La educación no presentó correlación con el nivel de conocimiento de la práctica. Se observó una asociación entre el tiempo del diagnóstico y la presencia de complicaciones, donde el $70,0 \%$ de la muestra tenía diabetes con complicaciones para un período $\geq 10$ años.

Por lo tanto, los esfuerzos educativos para facilitar el acceso a los conocimientos sobre el cuidado de los pies son esenciales para la prevención del pie diabético. 


\section{RESUMO}

O objetivo desta pesquisa foi identificar o conhecimento de pessoas com Diabetes Mellitus em relação aos cuidados com os pés, correlacionando o tempo de estudo com o número de respostas concordantes, bem como o tempo de diagnóstico com a presença de complicações crônicas.

Trata-se de estudo analítico, exploratório e transversal, realizado com 41 indivíduos, portadores de diabetes, atendidos em um ambulatório especializado de uma cidade do Triângulo Mineiro.

Os resultados mostraram déficit no conhecimento desta população sobre os cuidados podais, já que a média de acertos foi de 10,0 perante o total de 15 questões. A escolaridade não apresentou correlação com o nível de conhecimento acerca desta prática. Houve associação entre o tempo de diagnóstico e a presença de complicações, onde $70,0 \%$ da amostra com complicações tinham diabetes por período $\geq$ 10 anos.

Portanto, ações educativas que viabilizem o acesso ao conhecimento sobre os cuidados com os pés são essenciais para a prevenção do pé diabético.

\section{ABSTRACT}

The aim of this research was to identify the knowledge of people with Diabetes Mellitus in relation to foot care, correlating the study time with the number of concordants responses, as well as, the time of diagnosis with the presence of chronic complications.

It is analytical, exploratory and cross-sectional study, carried out with about 41 individuals with diabetes who attended an outpatient clinic of a city in Triangulo Mineiro.

The results showed a deficit in knowledge about the feet care of this population, since the average score was 10.0 in the total of 15 questions. Education was not correlated with the level of knowledge of the practice. There was an association between the time of diagnosis and the presence of complications, where $70.0 \%$ of the sample had diabetes with complications for a period $\geq 10$ years.

Therefore, educational efforts to provide access to knowledge about foot care are essential to preventing this sickness.

\section{INTRODUCCIÓN}

La Diabetes Mellitus (DM) es un síndrome caraterizado por problemas metabólicos derivados de defectos en la acción y secreción de la insulina ${ }^{(1)}$. En Brasil, su incidencia es de $7,6 \%$ a $12,0 \%$ entre los individuos de 30 a 69 años, siendo que $46,5 \%$ de los diabéticos desconocen el diagnóstico ${ }^{(1,2)}$.

Esta enfermedad se caracteriza como un grave problema de salud pública, ya que las complicaciones crónicas derivadas del ineficaz control glicémico son responsables de la reducción en la productividad laboral y resultan en alta morbimortalidad ${ }^{(3)}$. Estudios demuestran la relación directa entre la progresión de la enfermedad y la incidencia de complicaciones, siendo la prevalencia de enfermedad cardiovascular y neuropatía de $30,1 \%$ y $17,8 \%$ respectivamente ${ }^{(4)}$.

El Pie Diabético (PD) es la principal causa de hospitalización y amputación no traumática entre los diabéticos ${ }^{(5)}$ y se caracteriza por el desarrollo de ulceraciones en los miembros inferiores decurrentes de la neuropatía y vasculopatía periférica ${ }^{(6)}$. Su impacto es relevante, toda vez que entre $15,0 \%$ a $20,0 \%$ de las personas con DM desarrollarán úlceras podológicas, siendo responsables de aproximadamente 85,0\% de estas amputaciones ${ }^{(5)}$. Es importante resaltar que las ulceraciones son causadas 
por hábitos inadecuados en el cuidado con los pies, desencadenando alteraciones físicas y psicológicas que afectan la autoestima ${ }^{(2)}$.

La prevención del PD está relacionada a la orientación profesional sobre prácticas simples como el conocimiento de la sensibilidad de los pies y sus implicaciones, la correcta higiene e hidratación de los pies, la elección de los zapatos apropiados y el autoexamen de los pies ${ }^{(7)}$. Sin embargo, se sabe que el grado de educación interfiere en el desarrollo de esas habilidades y en el acceso a las informaciones pertinentes para el autocuidado ${ }^{(8)}$.

La identificación del nivel de conocimiento de los portadores de DM sobre el asunto permite dirigir acciones educativas que promuevan e incentiven prácticas adecuadas de cuidado. En Brasil, esta temática es incipiente, con escasez de estudios que midan el conocimiento acerca de estos cuidados.

Frente a esto, los objetivos de esta investigación fueron: identificar el conocimiento de los portadores de DM en relación a los cuidados con los pies; correlacionar el tiempo de educación con el conocimiento sobre los cuidados con los pies y asociar el tiempo de diagnóstico de laDM conlas complicacionespresentes.

\section{MATERIALES Y MÉTODOS}

Se trata de un estudio analítico, exploratorio y transversal, realizado en el Ambulatorio de Endocrinología de la Universidad Federal del Triángulo Minero, en la ciudad de Uberaba-MG, con convenio al Sistema Único de Salud (SUS). La recolección de datos fue realizada entre los meses de junio a agosto de 2011. El trabajo fue aprobado por el Comité de Ética en Investigación (CEI) de la Universidad sobre el protocolo n. 1829.

Los criterios de inclusiónfueron: diagnóstico previo deDM tipo 1, 2, LADA (Latent Autoimmune Diabetes of the Adult) o MODY (Maturity-Onset Diabetes of the Young), y pertenecer al grupo entre 19 a 59 años. Fueron excluidos aquellos con diagnóstico de DM gestacional, con edad $\leq 18$ años $o \geq 60$ años y que presentaban Incapacidad intelectual de comprensión o comunicación.

Fueron invitados a participar del estudio 48 individuos,después de la aplicación del Consentimiento Libre y Esclarecido. Así, la muestra estuvo compuesta por 41 diabéticos, ya que siete no aceptaron participar.

Para la recolección de datos fue utilizado un instrumento subdividido en tres partes:

a) Datos sociodemográficos (identificación, género, edad, procedencia, estado civil, educación y condiciones de vivienda);

b) Datos de salud (tipo de diabetes, medicación, complicaciones de la diabetes, hipertensión arterial, dislipidemia, orientaciones nutricionales, práctica de ejercicio físico, tabaquismo, historia de amputaciones y lesiones previas), consultados en el prontuario;

c) Cuestionario para evaluar el conocimiento del entrevistado en relación a los cuidados con los pies, compuesto por 15 preguntas de opción múltiple con una respuesta correcta basado en el Consenso Internacional sobre el Pie Diabético ${ }^{(9)}$. 
Para evaluación y adecuación, el mismo fue apreciado por dos especialistas en DM. Posteriormente, se realizó un pre-test con tres sujetos con diabetes acompañados por el referido ambulatorio.

Luego del término de cada entrevista, todos los individuos recibieron un folleto ilustrado y auto-explicativo confeccionado de acuerdo con referencial teórico especializado ${ }^{(9,10)}$, conteniendo los siguientes tópicos: ‘¿Qué es la Diabetes?', 'Conociendo sus Pies' y 'Cuidados con los Pies'. En este momento, la entrevistadora aclaraba acerca de los cuidados incorrectos realizados y otras dudas presentadas por los propios entrevistados.

Los datos fueron organizados y digitados en una planilla del programa Excel® en carácter de entrada doble, para posterior validación y verificación de los datos inconsistentes. El análisis estadístico fue realizado por medio del software SPSS versión 17.0.

Para el primer objetivo fue utilizada la estadística descriptiva, mientras que para atender al segundo objetivo, fue verificada inicialmente la normalidad de los datos por medio del test de Kolmogorov Smirnov, y posteriormente fue aplicado el test Correlación de Pearson ( $r$ ).

Ya en el tercer objetivo la variable tiempo de diagnóstico fue categorizada en: $<10$ años $y \geq 10$ años; siendo aplicado el test Chi-Cuadrado $(x 2)$. El nivel de significancia (a) fue de 0,05 y los tests fueron considerados significativos cuando $p<0,05.6$

Los valores de $p$ fueron interpretados suponiendo que esta casuística constituyó una muestra aleatoria simple de una población con características similares.

\section{Datos sociodemográficos y de salud}

Con relación a las variables sociodemográficas analizadas, se observó que 65,9\% (27) de los sujetos entrevistados pertenecían al género femenino, 65,9\% (27) presentaban la primaria incompleta y $56,1 \%$ (23) se declararon casados. La media de edad fue de 44,6 $\pm 11,9$ años, con extremos de 19 a 58 años.

Con respecto al municipio de origen, $70,7 \%$ (29) eran procedentes de Uberaba y los demás provenían de ciudades vecinas de la macro-región del Triángulo Sul.

La investigación de las condiciones de vivienda también mostró que 73,2\% (30) vivían en casa propia. Y que el tipo de piso presente en 65,9\% (27) de las residencias era de cerámica y madera, con el patio trasero total o parcialmente de tierra en $59,5 \%$ (22) de ellas.

El DM tipo 2 fue prevalente, alcanzando $73,2 \%$ (30) de los sujetos evaluados. $Y$ el tiempo de diagnóstico fue de 10,6 \pm 7,4 años, con variación de 0,3 a 28 años (Figura $1)$. 
Figura 1. Tiempo de diagnóstico en años identificado entre los portadores de DM. Uberaba/MG, 2011. 7

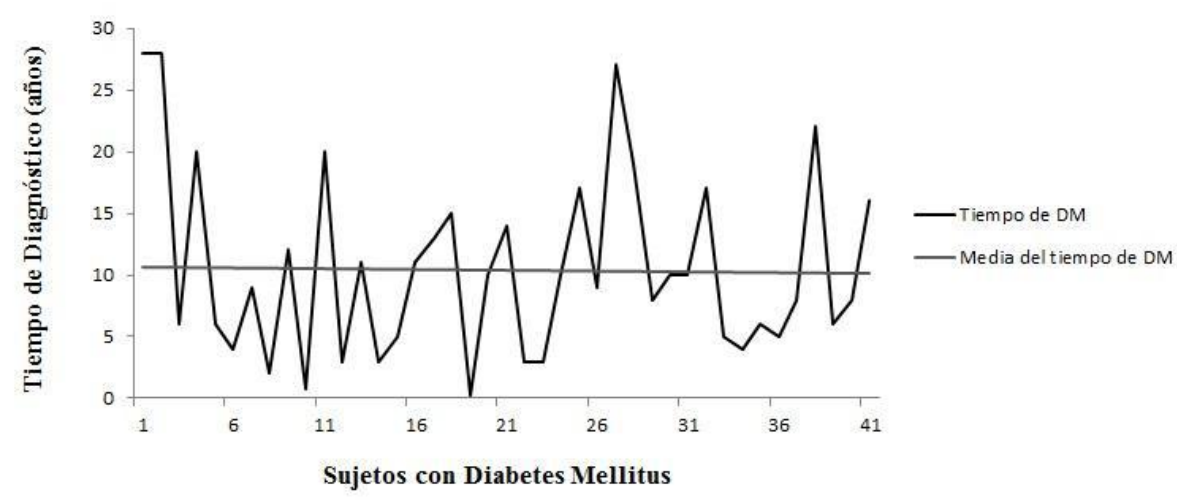

La medicación utilizada por 70,7\% (29) fue la insulina, aisladamente o en asociación con antidiabéticos orales.

Las complicaciones crónicas, como la neuropatía, retinopatía, nefropatía y enfermedad arterial coronario (EAC), estuvieron presentes en 43,9\% (18) de los entrevistados. De estos, 72,2\% (13) presentaban retinopatía diabética aislada, 0 concomitante a la neuropatía diabética.

En relación a la presencia de enfermedades, 63,4\% (26) presentaban hipertensión arterial sistémica (HAS) y la dislipidemia estuvo presente en $82,9 \%$ (34) de esta muestra.

La investigación de los hábitos comportamentales mostró que 56,1\% (23) no seguían las orientaciones nutricionales. Del mismo modo, 53,7\% (22) no practicaban ejercicio físico y apenas $14,6 \%$ (6) eran tabaquistas.

En relación a la amputación de miembros inferiores, esta estuvo ausente en 97,6\% (40) de los sujetos. La úlcera previa, a su vez, estuvo presente en 31,7\% (13), siendo que el local de tratamiento de esas lesiones podológicas para 69,2\% (9) fueron los servicios de salud.

\section{Cuidados con los pies}

Esta evaluación mostró que $100 \%$ (41) de los sujetos reconocen que los cuidados podológicos son importantes en la prevención del PD. Aunque, 82,9\% (34) no realizan el examen clínico anual o ni siquiera sabían de su importancia.

Al investigar sobre los cuidados en el domicilio, todos afirmaron realizar la higiene de los pies. Asociado a esto, 78,0\% (32) admitieron realizar la inspección diaria de los pies y espacios interdigitales. Cuando cuestionado sobre el auxilio del espejo en el examen de los pies, solo 7,3\% (3) relataron utilizarlo. El secado de los pies fue realizado por $90,2 \%$ (37) de los entrevistados, siendo que $87,8 \%$ (36) afirmaron secar la planta, dorso y entre los dedos. También, 75,6\% (31) relataron no usar zapatos apretados. Concomitante, la inspección de los zapatos estuvo presente en $87,8 \%$ (36) de ellos. Además de eso, 80,5\% (33) afirmaron no caminar descalzos. 
La investigación de los hábitos frente a las callosidades mostró que 51,2\% (21) realiza la remoción manual o no realizan cuidados apropiados para evitar lesiones. El cuidado con las uñas siguió este padrón, pues 53,7\% (22) reconocieron que las cortan de forma inapropiada. La hidratación incorrecta fue otro punto relevante, pues 53,7\% (22) hidrataban planta, dorso y espacios interdigitales.

Por fin, el apoyo familiar fue considerado esencial por todos los entrevistados. De estos, 73,2\% (30) piden ayuda cuando no consiguen realizar los cuidados solos.

Acerca del conocimiento en relación a los cuidados con los pies el total para las preguntas correctas fue poco satisfactorio. Entre las 15 preguntas evaluadas, la media de aciertos fue 10,0 $\pm 2,3$, con variación de 6 a 14 (Figura 2).

Figura 2. Número de respuestas concordantes y discordantes acerca de los cuidados con los pies, según los sujetos con DM. Uberaba/MG, 2011.

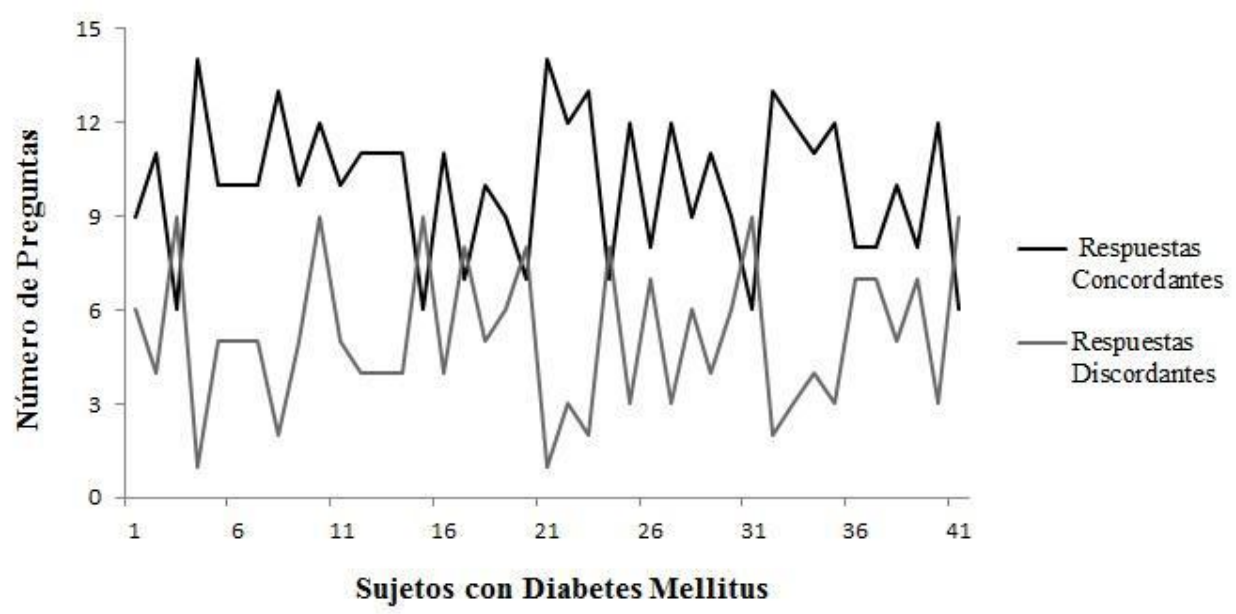

Además de eso, no fue significativa la correlación entre el tiempo de estudio en años y el número de respuestas concordantes acerca del conocimiento de los cuidados con los pies $(r=0,134 ; \rho=0,402)$.

Con todo, hubo asociación significativa entre la progresión del tiempo de diagnóstico y la presencia de complicaciones crónicas (Tabla I), donde 70,0\% (14) de los entrevistados con estas alteraciones presentaban DM por período mayor o igual a 10 años de enfermedad $\left(x^{2}=10,798 ; \rho=0,001\right)$.

Tabla I. Asociación entre el tiempo de diagnóstico y la presencia de complicaciones crónicas identificadas en los sujetos con DM. Uberaba/MG, 2011.

\begin{tabular}{|c|c|c|c|c|c|c|}
\hline \multirow{3}{*}{ Complicaciones DM } & \multicolumn{4}{|c|}{$D M \geq 10$ años } & \multirow{2}{*}{\multicolumn{2}{|c|}{ Total }} \\
\hline & \multicolumn{2}{|c|}{ Si } & \multicolumn{2}{|c|}{ No } & & \\
\hline & $\mathrm{n}$ & $\%$ & $\mathrm{n}$ & $\%$ & $\mathrm{n}$ & $\%$ \\
\hline $\mathbf{S i}$ & 14 & 70,0 & 4 & 19,0 & 18 & 43,9 \\
\hline No & 6 & 30,0 & 17 & 81,0 & 23 & 56,1 \\
\hline Total & 20 & 100,0 & 21 & 100,0 & 41 & 100,0 \\
\hline
\end{tabular}


La progresión de curso lento del DM hace que las complicaciones podológicas sean negligencias o desconocidas por varios individuos que conviven con esta enfermedad. De este modo, se hace necesario identificar el nivel de conocimiento relacionado con los cuidados correctos de los pies y otras características de esta población que puedan contribuir a la prevención de las complicaciones podológicas de la diabetes.

En este estudio, la prevalencia del género femenino fue también encontrada en otros realizados con sujetos que presentaban $\mathrm{DM}^{(3,11,12)}$.

Del mismo modo, la media de edad de los entrevistados fue semejante a la encontrada en reciente evaluación sobre los cuidados con los pies, la cual fue de 44 años ${ }^{(13)}$.

En relación a la educación, la mayoría de los sujetos presentaba primer grado incompleto, siendo convergente con el porcentaje en otro servicio de salud que realizó investigación semejante, el cual fue de 65,9\%(12).

Conocer el grado de instrucción de las personas con DM es fundamental para el planeamiento de las acciones de enfermería que miren a favorecer la educación en salud referente a la sensibilidad de la prevención del PD.

Las condiciones de vivienda también deben ser investigadas, ya que son importantes en el planeamiento del cuidado. La reducción de la sensibilidad periférica implicará en adaptaciones en el ambiente doméstico de modo a prevenir traumas de miembros inferiores.

Estos datos se mostraron divergentes en cuanto al tipo de piso y patio cuando comparados a los de otra investigación que encontró solo 15,6\% uso de cerámica y madera, y $40,6 \%$ con el patio total o parcialmente de tierra ${ }^{(12)}$.

En relación a la caracterización de la enfermedad, la DM tipo 2 fue prevalente entre la mayoría de los entrevistados en el ambulatorio. Investigaciones para identificar la aplicación de los cuidados podológicos y los factores de riesgo para el PD, encontraron porcentajes entre $79,5 \%$ y $86,0 \%$ para el tipo 2 de la diabetes ${ }^{(2,14)}$.

El tiempo de diagnóstico de la DM presentó variación considerable entre los entrevistados, tendiendo para un período de 10 años de enfermedad. Entre tanto, esta variable presentó valores entre 12,5 a 14,1 años en otros estudios (15,16). La delimitación del grupo de edad y el diagnóstico más tardío en la DM tipo 2 pueden haber contribuido a esta diferencia.

Del mismo modo, la insulina fue el tratamiento de elección en más de $70,0 \%$ de los entrevistados. Aún, los antidiabéticos orales parecen ser prevalentes en el manejo de la enfermedad en Brasil y en otros países, presentando porcentajes de elección entre $64,9 \%$ a $84,0 \%{ }^{(2,14,17,18)}$.

Una mayor incidencia de la resistencia a la insulina y agravamiento de la enfermedad podrían ser presumidos, ya que la media del tiempo de diagnóstico de la DM fue alto en este estudio. Sin embargo, investigaciones de laboratorio serían requeridas para confirmar esta inferencia. Además de eso, evaluación desarrollada en otro ambulatorio de endocrinología apuntó la insulina como medicación utilizada por 71,4\% de los sujetos de aquel servicio ${ }^{(16)}$. 
La presencia de complicaciones crónicas se dio en menos de la mitad de los entrevistados. Sin embargo, más de $71,0 \%$ de estos presentaban compromiso de la visión debido a la DM. Esta prevalencia fue semejante al encontrado en un programa desarrollado para prevención del PD, el cual fue de $71,6 \%{ }^{(7)}$.

La HAS y la dislipidemia, a su vez, estuvieron presentes en más de $60,0 \%$ y $80,0 \%$ de los sujetos, respectivamente. Resultado coincidente con varios trabajos, donde la prevalencia de la hipertensión presentó variación entre $68,2 \%$ y $84,6 \%{ }^{(1-3,7,17,19)}$. A su vez, la dislipidemia también se dio en $81,1 \%$ de aquellos que presentaban concomitante neuropatía periférica ${ }^{(20)}$.

Estos hallazgos pueden ser reflejo, nuevamente, de dificultades en el control glicémico, progresión de la DM y la no adopción de hábitos saludables, como alimentación, práctica de ejercicio físico, cese del tabaquismo.

La literatura demuestra que casi $69,0 \%$ de los sujetos con diabetes adhieren a las mudanzas de hábitos alimentarios y en torno de $65,0 \%$ practican algún tipo de actividad física ${ }^{(17)}$.

Se sabe que esas mudanzas colaboran en el control de la presión arterial, reducción del colesterol y triglicéridos, y consecuentemente en la prevención de complicaciones micro y macro vasculares. Ya que la presencia de la hipertensión y el tiempo de DM son factores de riesgo para enfermedad vascular periférica (EVP), cerebrovascular y retinopatía $^{(14)}$.

A pesar de la baja incidencia de amputaciones de miembros inferiores en esta investigación, la presencia de neuropatía favorece el aumento del riesgo para el desarrollo de ulceraciones y consecuentemente, amputaciones.

La ausencia de la sensibilidad periférica compromete la circulación podológica debido al perjuicio de la inervación de pequeños músculos de los pies. Consecuentemente, esto lleva a alteraciones en la distribución del peso durante la deambulación favoreciendo la formación de callosidades, necrosis isquémica en el tejido subcutáneo adyacente $y$, por fin, perjuicio en el reconocimiento de ulceraciones iniciales ${ }^{(21)}$.

Este acto està en consonancia con otros relatos en la literatura al demostrar que más de $60,0 \%$ de las amputaciones de miembros inferiores en Brasil son ocasionadas por úlceras previas ${ }^{(5,20,22)}$.

Por lo tanto, acciones educativas nacionales que sensibilicen la importancia del autocuidado y examen clínico anual de los pies son esenciales, ya que el riesgo de lesiones podológicas es estimado en $15,0 \%{ }^{(7)}$ durante la evolución de la enfermedad. La investigación del conocimiento en relación a los cuidados con los pies en el domicilio mostró la concienciación de los sujetos de este estudio sobre la importancia de la higienización e inspección diaria de los pies. Otro relato mostró que 94,5\% y $81,8 \%$ de las personas con DM realizaban estos cuidados ${ }^{(17)}$, respectivamente.

Sin embargo, el uso del espejo en el examen complementario todavía es pequeño. Tal vez esto sea reflejo de las características de la muestra, constituida por adultos jóvenes. Aún así, este instrumental debe formar parte del autoexamen ${ }^{(19)}$, para ayudar a superar las limitaciones físicas. 
El secado correcto de los pies es practicado por casi $90 \%$ de los diabéticos. Esta práctica fue superior a la encontrada en otros servicios públicos de salud donde esta proporción osciló entre 70,9\% (17) y 83,7\% (19).

Padrón semejante fue observado en relación a los cuidados con los calzados, donde la mayoría afirmó evitar el uso de zapatos que puedan causar lesiones podológicas, así como los hábitos de examinarlos y no caminar descalzo. Todavía, debido al alto impacto de estas acciones en la prevención del PD, estos cuidados deberían ser realizados por todos los entrevistados.

La literatura apunta a una prevalencia mayor de $97,0 \%$ en relación a la preferencia por calzados que se adecúan a las características anatómicas de los pies entre estos sujetos ${ }^{(3)}$ y mayor de $84,0 \%$ a no andar descalzo ${ }^{(7,23)}$. En contrapartida, revela práctica inferior de la inspección de los zapatos ${ }^{(7,12)}$ cuando comparado con este estudio.

Estos hábitos deben ser incentivados, principalmente, debido al riesgo aumentado para lesiones en aquellos pies cuya sensibilidad táctil, dolorosa ${ }^{(12)}$ y cicatrización están comprometidas.

Los cuidados correctos con callosidades, uñas e hidratación de los pies deben ser estimulados, ya que la mayoría mostró desconocerlos. Esta realidad fue perceptible en otros relatos, los cuales demostraron la prevalencia de la remoción manual de callos, corte inapropiados de las uñas e hidratación entre los espacios interdigitales $^{(17,19,23)}$.

Promover la adhesión a estas prácticas es un desafío en Brasil, ya que tanto la atención primaria como la secundaria en salud carecen de programas especializados para prevención, educación y acompañamiento regular de los pies de las personas con diabetes.

La familia, como significativa institución social, se torna en un eslabón importante en este contexto ya que es responsable der la transmisión de valores y culturas individuales que reflejan en sus miembros. De esta forma, se caracteriza como importante centro de apoyo ante el cuidado en salud ${ }^{(24)}$.

En el presente trabajo el número de preguntas correctas acerca de los cuidados de los pies presentó considerable variación, haciéndose necesarias intervenciones como conferencias, 14 folletos auto-explicativos debido al desconocimiento de la forma adecuada del corte de las uñas o importancia del examen clínico anual, por ejemplo.

A pesar de que el tiempo de estudio no interfiere en el nivel de conocimiento entre los entrevistados de este servicio de salud, es importante resaltar que la menor educación $^{(8,9,13,18)}$ y el nivel socioeconómico ${ }^{(13)}$, pueden influir en la adquisición de informaciones respecto de los cuidados adecuados en los pies o búsqueda precoz de ayuda profesional ${ }^{(8,16)}$ en presencia de alteraciones podológicas $u$ otras complicaciones.

La asociación entre el tiempo de diabetes y la presencia de complicaciones crónicas encontradas en este estudio, también fue observada en la literatura. Con la progresión de la enfermedad el riesgo para la enfermedad cardiovascular, retinopatía ${ }^{(4)}$, 
neuropatía ${ }^{(4,14)}$ y EVP ${ }^{(14)}$ presentó significativo aumento en relación a aquellos con menor período de diagnóstico de la enfermedad.

Frente a esto, se nota la relevancia de las acciones de educación en salud junto a los individuos con $\mathrm{DM}$ en el ámbito de la atención primaria y secundaria, con el propósitoando de disminuir el impacto de estas disparidades en la incidencia del PD y otras complicaciones crónicas.

\section{CONCLUSIÓN}

El conocimiento de los cuidados de los pies es esencial en la prevención del PD, ejerciendo el equipo de salud papel fundamental en el proceso de sensibilidad de los de esta población.

Es perceptible un déficit en el conocimiento de los cuidados podológicos entre los sujetos evaluados en el presente servicio de salud, a pesar de la educación no mostrarse factor limitante cuando correlacionada con el número de respuestas correctas sobre el autocuidado. Sin emb argoo, el tiempo de diagnóstico fue proporcional a la presencia de complicaciones crónicas.

De este modo, acciones educativas que hacen viable el acceso al conocimiento sobre los cuidados de los pies y la evaluación anual de los pies, son esenciales para la prevención del PD y promoción de la calidad de vida de las personas con diabetes acompañadas en este servicio de salud.

\section{REFERENCIAS}

1.Morais GFC, Soares MJGO, Costa MML, Santos IBC. O diabético diante do tratamento, fatores de risco e complicações crônicas. Rev Enferm UERJ. 2009; 17(2):240-5.

2. Carvalho RDP, Carvalho CDP, Martins DA. Aplicação dos cuidados com os pés entre portadores de diabetes mellitus. Cogitare Enferm. 2010; 15(1):106-9.

3. Teixeira CJ, Oliveira ACP, Bazotte RB, Batista MR. Pé diabético: perfil metabólico e socioeconômico de pacientes atendidos pelo laboratório de ensino e pesquisa da Universidade Estadual de Maringá. Arq Ciênc Saúde. 2010; 14(2):125-32.

4. Liu Z, Fu C, Wang W, Xu B. Prevalence of chronic complications of type 2 diabetes mellitus in outpatients - a cross-sectional hospital based survey in urban China. Health Qual Life Outcomes [Internet]. 2010 [Cited 2011 Dec 19]; 8:62. Available from: http://www.hqlo.com/content/8/1/62.

5. Rezende KF, Ferraz MB, Malerbi DA, Melo NH, Nunes MP, Pedrosa HC, et al. Direct costs and outcomes for inpatients with diabetes mellitus and foot ulcers in a developing country: The experience of the public health system of Brazil. Diabetes Metab Syndr. 2009; 3(4):228-32.

6. Audi EG, Moreira RC, Moreira ACG, Pinheiro EFC, Mantovani MF, Araújo AG. Avaliação dos pés e classificação do risco para pé diabético: contribuições da enfermagem. Cogitare Enferm. 2011; 16(2): 240-6.

7. Anselmo MI, Nery M, Parisi MCR. The effectiveness of educational practice in diabetic foot: a view from Brazil. Diabetol Metab Syndr [Internet]. 2010; [Cited 2012 Nov 3]; 2:45. Available from: http://www.dmsjournal.com/content/2/1/45.

8. Nath C. Literacy and Diabetes Self-Management. Am J Nurs. 2007; 107(6): 43-9. 
9. Grupo de Trabalho Internacional sobre Pé Diabético. Consenso Internacional sobre Pé Diabético. Brasília (Brasil): Secretaria de Estado de Saúde do Distrito Federal; 2001.

10. Escola de Enfermagem de Ribeirão Preto [homepage na internet]. Ribeirão Preto: Universidade de São Paulo; c1998-2012 [cited 2011 May]. Feridas Crônicas: Pé Diabético; [aproximadamente 5 telas]. Disponível em: http://www2.eerp.usp.br/site/grupos/feridascronicas/index.php.

11. Bortoletto MSS, Haddad MCL, Karino ME. Pé diabético, uma avaliação sistematizada. Arq Ciênc Saúde UNIPAR. 2009; 13(1): 37-43.

12. Barbui EC, Cocco MIM. Conhecimento do clinte diabético em relação aos cuidados com os pés. Rev Esc Enferm USP. 2002; 36(1):97-103.

13. Desalu OO, Salawu FK, Jimoh AK, Adekoya AO, Busari AO, Olokoba AB. Diabetic foot care: self reported knowledge and practice among patients attending three tertiary hospital in Nigeria. Ghana Med J. 2011; 45(2): 60-5.

14. Al-Maskari F, El-Sadig M. Prevalence of risk factors for diabetic foot complications. BMC Fam Pract [Internet]. 2007 [Cited 2012 Nov 2]; 8:59. Available from: http://www.biomedcentral.com/1471-2296/8/59.

15. Laurindo MC, Recco DC, Roberti DB, Rodrigues CDS. Conhecimento das pessoas diabéticas acerca dos cuidados com os pés. Arq Ciênc Saúde. 2005; 12(2):80-4.

16. Pace AE, Ochoa-Vigo K, Caliri MH, Fernandes APM. O conhecimento sobre diabetes mellitus no processo de autocuidado. Rev Latinoam Enfermagem. 2006; 14(5): 728-34.

17. Rocha RM, Zanetti ML, Santos MA. Comportamento e conhecimento: fundamentos para prevenção do pé diabético. Acta Paul Enferm. 2009; 22(1):17-23.

18. Khamseh ME, Vatankhah N, Baradaran HR. Knowledge and practice of foot care in Iranian people with type 2 diabetes. Int Wound J. 2007; 4(4):298-302.

19. Ochoa-Vigo K, Pace AE. Prevenção de complicações nos pés de pessoas com diabetes mellitus: Proposta de cuidado. Rev Méd Hered. 2009; 20(2):77-88.

20. Porciúncula MVP, Rolim LCP, Garofolo L, Ferreira SRG. Análise de fatores associados à ulceração de extremidades em indivíduos diabéticos com neuropatia periférica. Arq Bras Endocrinol Metab. 2007; 51(7):1134-42.

21. Jeffcoate WJ, Harding KG. Diabetic foot ulcers. Lancet. 2003; 361(9368):1545-51. 22. Rezende KF, Nunes MAP, Melo NH, Malerbi D, Chacra AR, Ferraz MB. Internações por pé diabético: comparação entre o custo direto estimado e 0 desembolso do SUS. Arq Bras Endrocrinol Metab. 2008; 52(3):523-30.

23. Amaral AS, Tavares DMS. Cuidados com os pés: conhecimento entre pessoas com diabetes mellitus. Rev Eletr Enf. 2009; 11(4):801-10.

24. Moreira RC, Cruz CFR, Valsecchi EASS, Marcon SS. Vivências em família das necessidades de cuidados referentes à insulinoterapia e prevenção do pé diabético. Rev Gaúcha Enferm. 2008; 29(2):283-91.

ISSN 1695-6141

() COPYRIGHT Servicio de Publicaciones - Universidad de Murcia 